\title{
Transgenerational inheritance of promoter methylation changes in extrauterine growth restriction-induced pulmonary arterial pressure disorders
}

\author{
Lili Tang" ${ }^{1 \#}$, Ping Chen ${ }^{2 \#}$, Liu Yang ${ }^{3 \#}$, Jiyuan Liu ${ }^{4}$, Yuanfang Zheng ${ }^{2}$, Jincai Lin $^{2}$, Senhua Chen ${ }^{2}$, \\ Yinzhu Luo ${ }^{2}$, Yanyan Chen ${ }^{2}$, Xiaoying $\mathrm{Ma}^{2}$, Liyan Zhang ${ }^{2}$ \\ ${ }^{1}$ Department of Neonatology, Children's Medical Center Affiliated to Shanghai Jiaotong University School of Medicine, Shanghai, China; \\ ${ }^{2}$ Department of Neonatology, The Affiliated Fuzhou Children Hospital of Fujian Medical University, Fuzhou, China; ${ }^{3}$ Unimed Scientific Inc., Wuxi, \\ China; ${ }^{4}$ Fujian Medical University, Fuzhou, China \\ Contributions: (I) Conception and design: L Zhang; (II) Administrative support: L Zhang; (III) Provision of study materials or patients: L Tang; (IV) \\ Collection and assembly of data: P Chen; (V) Data analysis and interpretation: L Yang; (VI) Manuscript writing: All authors; (VII) Final approval of \\ manuscript: All authors. \\ \#These authors contributed equally to this work. \\ Correspondence to: Liyan Zhang. The Affiliated Fuzhou Children Hospital of Fujian Medical University, Fuzhou 350005, China. Email: liyanneo@163.com.
}

Background: This study aimed to investigate the influence of extrauterine growth restriction (EUGR) on pulmonary arterial pressure (PAP) and the transgenerational inheritance of promoter methylation changes in pulmonary vascular endothelial cells (PVECs) of 2 consecutive generations under EUGR stress.

Methods: After modeling, PAP values of F1 and F2 pups were investigated at 9-week-old. The methylDNA immune precipitation chip was used to analyze DNA methylation profiling. Differential enrichment peaks (DEPs) and regions of interest (ROIs) were identified, based on which Gene Ontology (GO) enrichment, Kyoto Encyclopedia of Genes and Genomes (KEGG) pathway, and reactome pathway enrichments were analyzed.

Results: The F1 male rats in the EUGR group had significantly increased PAP levels compared to the control group; however, this increase was not observed in female rats. Interestingly, in F2 female rats, the EUGR group had decreased PAP. In the X chromosome of the F1 males, there were 16 differential ROI genes in the F1 generation, while in F2 females, there were 86 differential ROI genes. Similarly, there were 105 DEPs in the F1 generation and 38 DEPs in the F2 generation. In combination with the 5 common ROIs and 14 common DEPs, 18 genes were regarded as the key candidate genes associated with hereditable PAP variation in the EUGR model. Enrichment analysis showed that synaptic and neurotransmitter relative pathways might be involved in the process of EUGR-induced PAH development. Among common DEPs, Smad1 and Serpine1 were also found in $102 \mathrm{PAH}$-associated genes in the MalaCards database.

Conclusions: Together, there is a transgenerational inheritance of promoter methylation changes in the $\mathrm{X}$ chromosome in EUGR-induced PAP disorders, which involves the participation of synaptic and neurotransmitter relative pathways. Also, attenuated methylation of Smad1 and Serpine1 in the promoter region may be a partial driver of $\mathrm{PAH}$ in later life.

Keywords: DNA methylation; pulmonary arterial pressure (PAP); epigenetics; extrauterine growth restriction (EUGR); transgenerational inheritance

Submitted Aug 17, 2021. Accepted for publication Oct 02, 2021.

doi: 10.21037/atm-21-4715

View this article at: https://dx.doi.org/10.21037/atm-21-4715 


\section{Introduction}

Extrauterine growth restriction (EUGR) can lead to undernutrition and premature infants, and it is a significant risk factor for a variety of lung or cardiovascular diseases. Under EUGR stress, there are obstructive vascular lesions of the distal pulmonary arteries, which may lead to pulmonary arterial pressure (PAP) disorders, especially pulmonary arterial hypertension (PAH). Although the incidence of $\mathrm{PAH}$ is low, it is of high mortality. It is associated with heterogeneous etiologies and distinct molecular mechanisms, including abnormal migration and proliferation of endothelial and smooth muscle cells (1). Pulmonary artery smooth muscle cells (PASMCs) are known model cells of PAH, and in these cells, MAPK signaling pathway may play a pathogenic role (2). Moreover, chronic hypoxia causes a metabolic disorder and the Warburg effect in PASMCs (3). Features including stress to endothelial cells and their corresponding apoptosis are noticed in $\mathrm{PAH}$ individuals (4). As known studies and our pilot observation have shown, pulmonary vascular endothelial cell (PVEC) injury is one of the pathogeneses of PAH (e.g., decreased Notch1 in PVECs) (5-7).

Previously, we found that alterations in phenotype can be transmitted to offspring. We reported that hypoxic $\mathrm{PAH}$ induced by intrauterine growth restriction (IUGR) was accompanied by epigenetic regulation of different genes (8). Traditionally, PAH models can be induced by monocrotaline (9), but EUGR- and IUGR-induced PAH have not been well studied. Transgenerational inheritance is used to describe the non-sequence-based effects that can be transmitted to the next generation. Known mechanisms include self-sustaining feedback loops, chromatin-based mechanisms, noncoding and coding RNA, and other nonepigenetic mechanisms. Recent epigenetic studies have confirmed that the transgenerational effect can be found in generations not exposed to the initial environment. So far, DNA methylation is the best-studied chromatinbased mechanism. Epigenetic modifications, especially DNA methylation, are key genomic regulatory processes in developmental origins. This postnatal stress can include epigenetic modification of genes linking to pulmonary vascular regulation in later life, and pulmonary vascular changes can be passed on to the offspring. So far, very few DNA-methylation studies have thoroughly characterized the pathogenesis of PAH (10). Therefore, the effects of EUGR on DNA methylation reprogramming have not been elucidated. In particular, the common changes in DNA methylation in the stressed generation and its offspring are unknown.

In the present study, we established an EUGR rat model and observed the PAH phenotype in 2 consecutive generations. We noticed that the stressed pups and their offspring had different phenotypes, and some promoter methylation profiling in the PVECs can be transmitted.

We present the following article in accordance with the ARRIVE reporting checklist (available at https://dx.doi. org/10.21037/atm-21-4715).

\section{Methods}

\section{EUGR rat model}

Pregnant Sprague-Dawley female rats were used to establish the EUGR model. All animals were obtained from Zhejiang University Laboratory Animal Center. Experiments were performed under a project license (zju201305-1-02-043) granted by The Affiliated Fuzhou Children Hospital of Fujian Medical University, in compliance with the institutional guidelines for the care and use of animals. Healthy pregnant rats (weight 30-40 g) were kept at $23{ }^{\circ} \mathrm{C}$ until giving birth. Within $12 \mathrm{~h}$ of birth, pups were randomly assigned to 2 groups: 10 pups of the control group were sent into a litter, and 20 pups of the EUGR group were placed into a litter (with a 1:1 male-tofemale ratio). Pups were weighed every day until day 21 . If a pup in the EUGR litter weighted the control group's last 10th percentile, it was defined as a successful EUGR individual. The PAP was measured until 9 weeks. Briefly, a PE50 catheter was inserted into the right atrium through the right jugular vein. The catheter was finally sealed to the right ventricle (an additional distance of $10 \mathrm{~mm}$ was left in the pulmonary artery). The hemodynamic values were automatically calculated by a physiological data acquisition system (Acqknowledge4 MP150 Biopac System Inc., Goleta, CA, USA). Next, each rat's lung was distended by the infusion of phosphate buffered saline (PBS) into the trachea. Afterwards, PVECs were isolated from fresh lungs. The flow chart of this experiment is shown in Figure 1. The pregnant female rats were regarded as $\mathrm{F} 0$ generation, the stressed generation was regarded $\mathrm{F} 1$, and the pups of $\mathrm{F} 1$ were regarded as F2. The F1 control female rats mated with either F1 control males or F1 EUGR males.

\section{Methyl-DNA immune precipitation chip}

For each group, 3 independent samples were used for chip analysis. According to the PAH phenotype, $3 \mathrm{~F} 1$ males in 


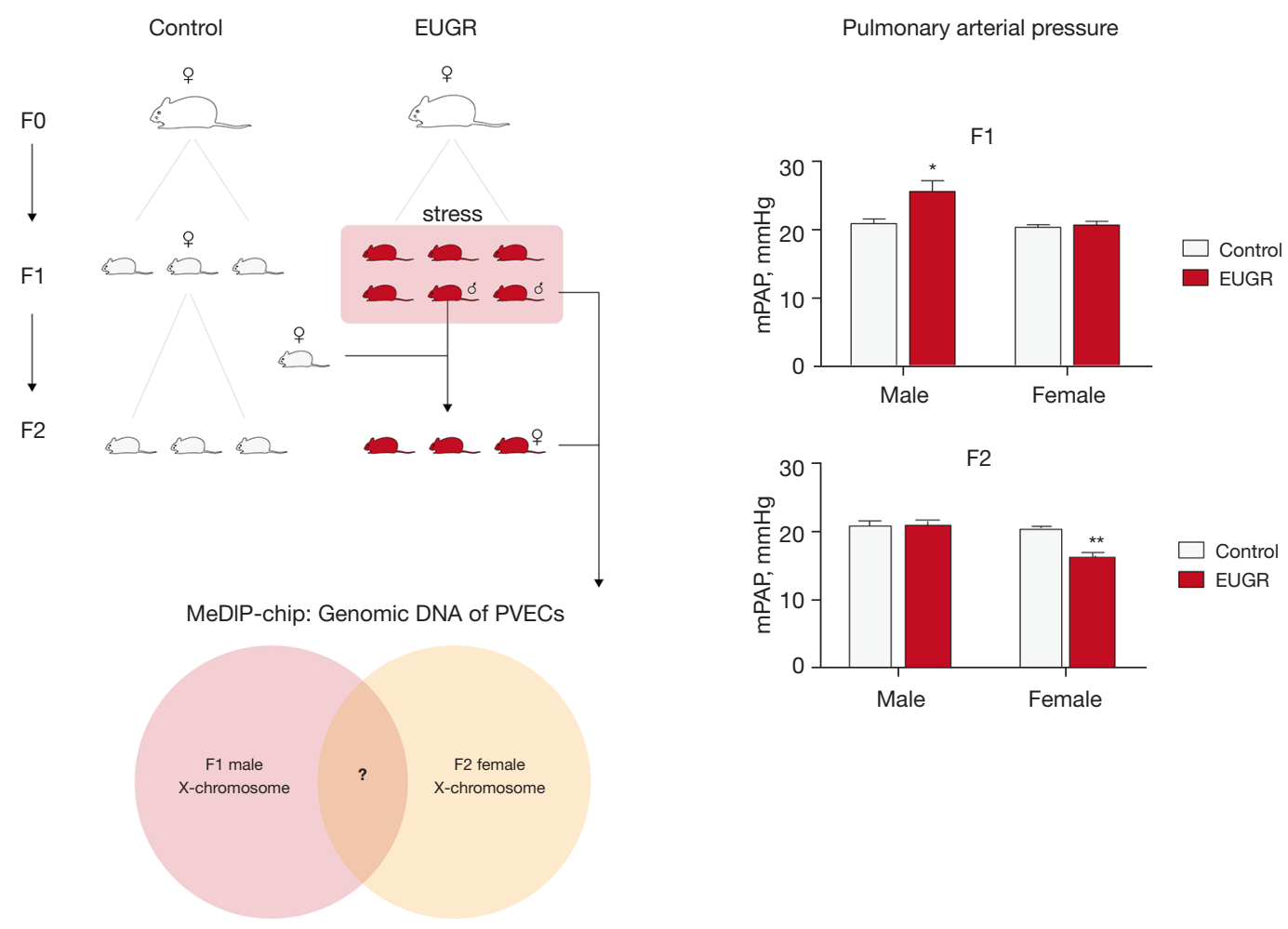

Figure 1 The flow chart of investigation on 2 generations and the phenotype changes. The F1 male rats but not female rats in the EUGR group have significantly increased $\mathrm{PAP}$ versus the control group. In $\mathrm{F} 2$ female rats, the EUGR group has decreased $\mathrm{PAP}$. ${ }^{*} \mathrm{P}<0.05$; ${ }^{* *} \mathrm{P}<0.01$. EUGR, extrauterine growth restriction; PAP, pulmonary arterial pressure.

the control group were compared with $3 \mathrm{~F} 1$ males in the EUGR group, and $3 \mathrm{~F} 2$ females in the control group were compared with $3 \mathrm{~F} 2$ females in the EUGR group. First, PVECs were isolated by magnetic-activated cell sorting. Fresh lungs were sliced and incubated with collagenase A. The suspension was centrifuged and washed 3 times [by PBS with $0.5 \%$ bovine serum albumin (BSA) and $2 \mathrm{mmol} / \mathrm{L}$ ethylenediaminetetraacetic acid (EDTA)]. The final pellets were resuspended as $10^{7}$ cells per $100 \mathrm{~mL}$. Cells were incubated with phycoerythrin (PE)-labeled mouse antirat CD31 antibody (PECAM-1, BD Pharmingen, San Diego, CA, USA) for $15 \mathrm{~min}$ at $4{ }^{\circ} \mathrm{C}$. After washing, anti-PE MicroBeads (Miltenyi Biotec, Bergisch Gladbach, Germany) were added to the cell suspension, and they were inoculated at $4{ }^{\circ} \mathrm{C}$ for $15 \mathrm{~min}$. The PVECs linked to PECAM- 1 antibodies were purified. According to the official protocol, the genomic DNA of PVECs was extracted, and DNA methylation status was determined using the MeDIP-chip kit (CapitalBio, Beijing, China). Briefly, DNA samples were digested into 200 to $1,000 \mathrm{bp}$ fragments. The antibody against 5-methylcytidine (Abcam, Cambridge, MA, USA) was used to label the methyl-DNA. In this study, we mainly focused on the promoter methylation changes. Therefore, the Plus-Ref-Seq Promoter Array (BD Pharmingen, USA) kit was used, which covered 15,287 promoters. The RefSeq genes were derived from the University of California Santa Cruz (UCSC) refFlat files. The raw data of microarray dots were analyzed using NimbleScan (version 2.5; Roche, Basel, Switzerland). Ratios of $\mathrm{Cy} 5 / \mathrm{Cy} 3$ signals were calculated for high-quality hybridization dots. These ratios were transformed to the normalized $\log 2$-ratios. The peakfinding algorithm in the NimbleScan software was used for peak detection.

\section{Region of interest and differential enrichment peak analysis}

Differentially methylated region of interest (ROI) in promoter was compared between 2 groups using mean $\log 2$-ratio across defined regions. The $\log 2$-ratio value 
of the control minus EUGR group was calculated as a differential value of methylated ROI. The difference between the 2 groups was assessed, and the $\mathrm{P}$ value was calculated using the Wilcoxon test. Differential enrichment peaks (DEPs) were identified in the promoter which contained all identified DEPs overlapping the promoter region of the relevant transcript (from $-3,880$ to $970 \mathrm{bp}$ ). The DEPs were calculated by the peak-score, namely the average $-\log 10$ (P value) from probes within the peak. All DEPs between groups were extracted. Additionally, given the phenotype was changed in F1 male and F2 female rats, it was reasonable to probe the inheritable promoter methylation changes in chromosome $\mathrm{X}$. The common ROIs and DEPs in F1-generation and F2-generation samples were pooled for enrichment analysis.

\section{Enrichment analysis}

Gene Ontology (GO) functional enrichment and Kyoto Encyclopedia of Genes and Genomes (KEGG) pathway enrichment were analyzed based on differentially methylated genes (in DEPs or ROIs). The enrichment $\mathrm{P}$ value of the pathway ID was calculated based on Fisher's exact test. The WebGestalt tool (WEB-based Gene Set Analysis Toolkit; http://www.webgestalt.org) was used to perform GO, KEGG, and reactome pathway enrichments.

\section{Statistical analysis}

Statistical analyses were performed using $\mathrm{R}$ packages for identifying DEPs or ROIs, as well as the WebGestalt tool for enrichment analysis (and multiple hypothesis tests were performed to adjust the $\mathrm{P}$ value calculation). The $\mathrm{P}$ values less than 0.05 were considered statistically significant.

\section{Results}

\section{Hereditable DEPs and ROIs in 2 generations}

Since the healthy pregnant rats (weight $30-40 \mathrm{~g}$ ) gave birth, we observed the phenotype changes of 2 generations. As shown in Figure 1, the F1 male rats in the EUGR group had significantly increased PAP compared to the control group $(\mathrm{P}<0.05)$. However, this increase was not observed in female rats. Interestingly, in F2 female rats, the EUGR group had decreased PAP $(\mathrm{P}<0.01)$. Based on this finding, it was reasonable to probe the inheritable promoter methylation changes in Chromosome X. The methylation assay was performed on PVECs isolated from 9-weekold male rats ( $\mathrm{n}=3$ in each group) of the $\mathrm{F} 1$ generation and female rats of the $\mathrm{F} 2$ generation ( $\mathrm{n}=3$ in each group). In the $\mathrm{X}$ chromosome of $\mathrm{F} 1$ males, there were 16 differential ROI genes in the F1 generation (EUGR vs. Control), including 9 hypermethylation ROIs and 7 hypomethylation ROIs (Figure $2 A$ ). In the $\mathrm{X}$ chromosome of $\mathrm{F} 2$ females, there were 86 differential ROI genes, including 60 hypermethylation ROIs and 7 hypomethylation ROIs (Figure 2B). Between the 16-ROI set of F1 generation and the 86-ROI set of the F2 generation, 5 were found in common (Figure 2C). Similarly, there were 105 DEPs in the F1 generation (EUGR vs. Control) and 38 DEPs in the F2 generation (EUGR vs. Control), between which there were 14 in common (Figure 2D). Combined with the 5 common DOIs and 14 common DEPs, they had a common gene (Ptges3l1) and their union set had 18 genes, which were regarded as the key candidate genes associated with hereditable PAP variation in the EUGR model (Figure 2E). Besides Ptges3l1, key candidates included Rhox2, Slc7a3, Slitrk2, Syn1, Kcnd1, Tspyl2, Sts, Taf9b, Ngfrap1, Usp26, Kcne1l, RGD1565983, Tsx, RGD1562502, Bhlbb9, Bex2, and MGC114520. Theoretically, these genes may participate in the pathologic process of PAH induced by EUGR.

\section{Enrichment analysis}

First, based on DEPs and ROIs in the X chromosome, the 18 candidate genes were used for GO functional enrichment, KEGG pathway enrichment, and reactome pathway enrichment. As shown in Figure $3 A$, for GO enrichments, the covered genes were classified into 3 categories: biological process (BP) terms (red), cellular component (CC) terms (blue), and molecular function (MF) terms (green); however, no significantly enriched GO terms were found. When using the raw $\mathrm{P}$ value for screening, the following KEGG pathways were enriched: basal transcription factors, arachidonic acid metabolism, steroid hormone biosynthesis, herpes simplex infection, and metabolic pathways (Figure $3 B$ ). Similarly, using the raw $\mathrm{P}<0.05$ as a threshold, the enriched reactome pathways were as follows: phase 1-inactivation of fast $\mathrm{Na}+$ channels, aryl hydrocarbon receptor signaling, the activation of arylsulfatases, HSF1 activation, serotonin neurotransmitter release cycle, dopamine neurotransmitter release cycle, attenuation phase, receptor-type tyrosineprotein phosphatases, Ub-specific processing proteases, and neuronal system (Figure 3C). The above pathways have 
A
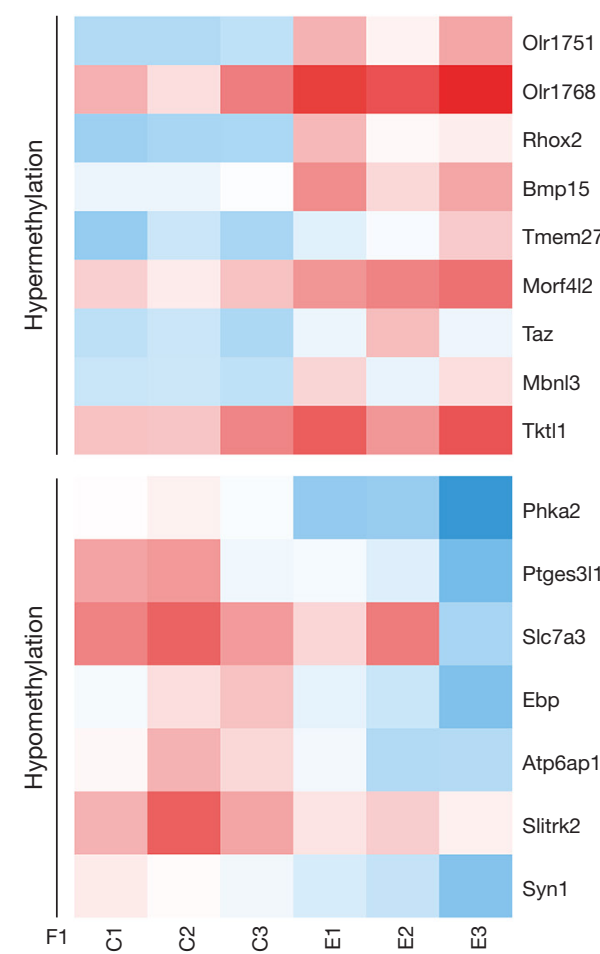

C

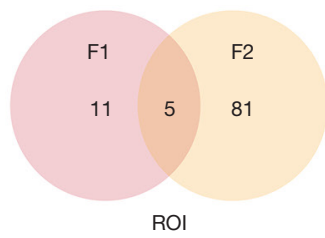

D

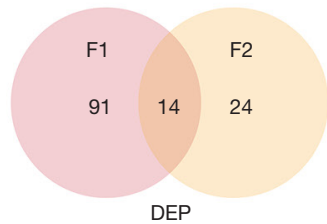

B
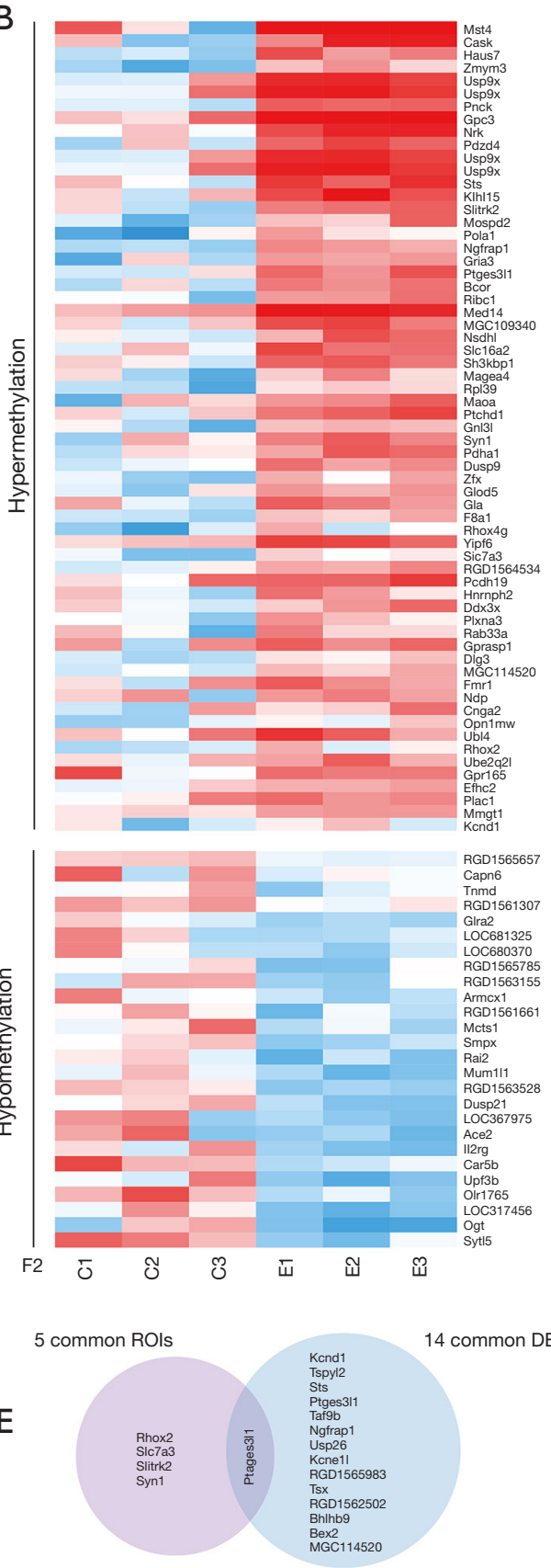

14 common DEPs

Together 18 key genes in chromosome $\mathrm{X}$

Figure 2 Major findings of DNA methylation changes in the X chromosome of PVECs. (A) In the X chromosome of F1 males, there are 16 differential ROI genes in the F1 generation (EUGR $v s$. Control), including 9 hypermethylation ROIs and 7 hypomethylation ROIs; (B) in the $\mathrm{X}$ chromosome of $\mathrm{F} 2$ females, there are 86 differential ROI genes, including 60 hypermethylation ROIs and 7 hypomethylation ROIs. (C) Between the 16-ROI set of F1 generation and the 86-ROI set of F2 generation, there are 5 common ROIs. (D) there were 105 DEPs in F1 generation and 38 DEPs in F2 generation, between which there are 14 common DEPs. (E) In combination with the 5 common DOIs and 14 common DEPs, they had a common gene Ptges3l1, and these 18 genes were regarded as the key candidate genes associated with hereditable PAP variation in the EUGR model. PVECs, pulmonary vascular endothelial cells; ROI, region of interest; EUGR, extrauterine growth restriction; DEPs, differential enrichment peaks; PAP, pulmonary arterial pressure. 
A

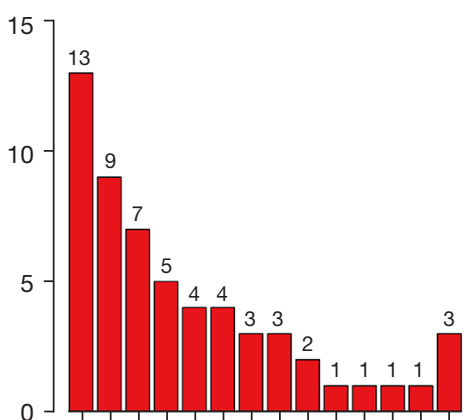

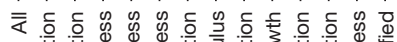

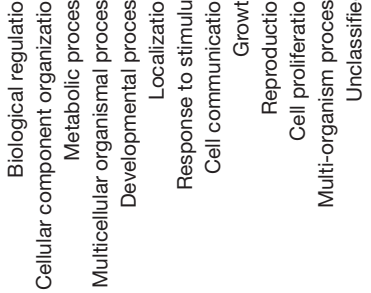

15

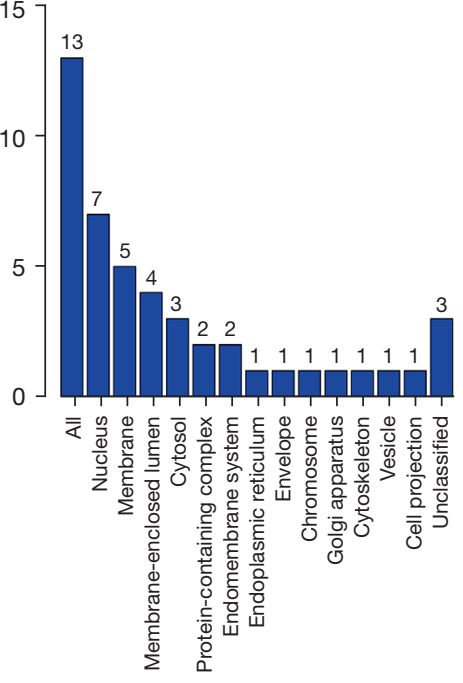

15

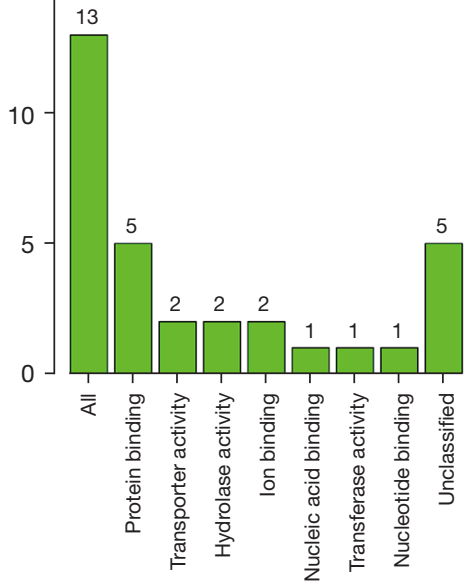

B

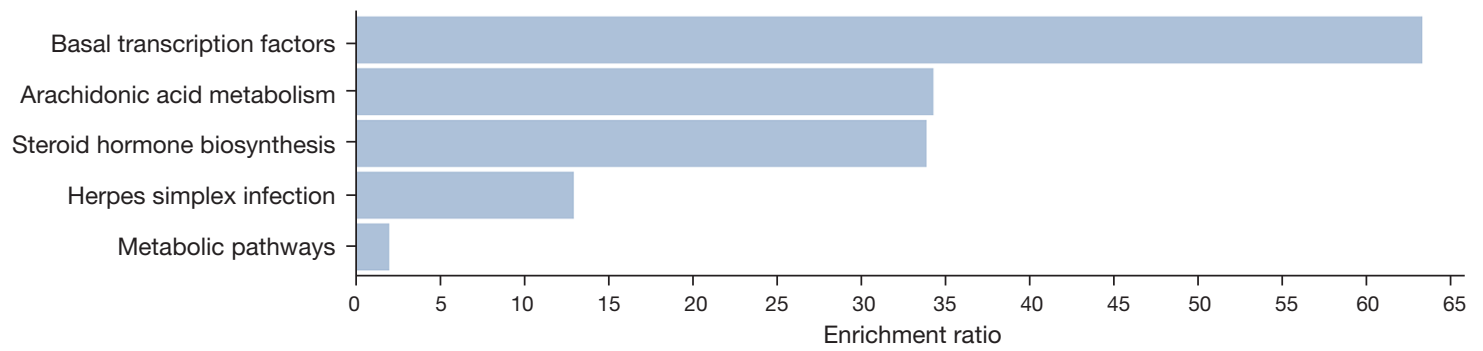

C

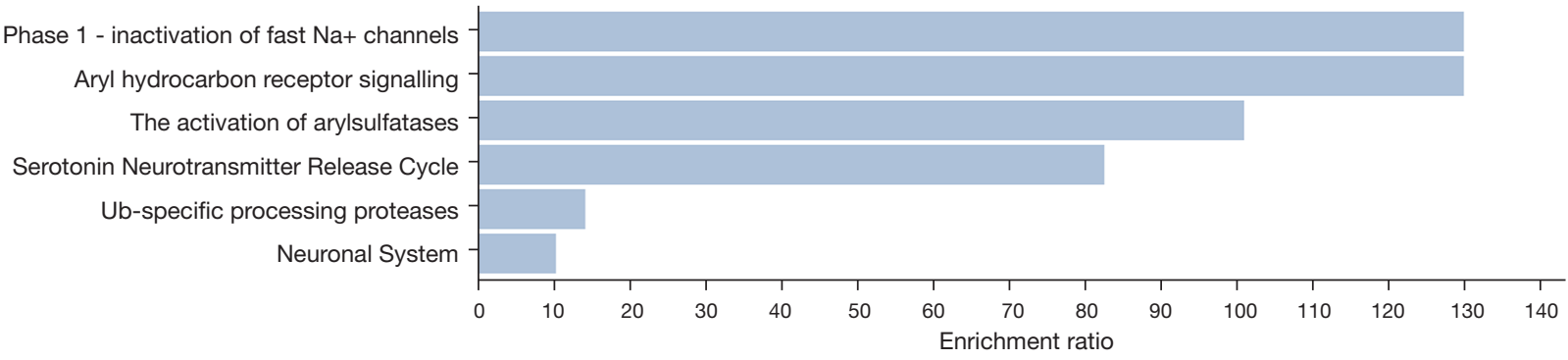

Figure 3 Enrichment analysis of 18 candidate genes (limited in the X-chromosome). (A) The covered genes in 3 categories: BP terms (red), CC terms (blue), and MF terms (green). No significantly enriched GO terms are found; (B) enriched KEGG pathways: Basal transcription factors, Arachidonic acid metabolism, Steroid hormone biosynthesis, Herpes simplex infection, and Metabolic pathways; (C) enriched reactome pathways: Phase 1-inactivation of fast $\mathrm{Na}^{+}$channels, Aryl hydrocarbon receptor signaling, The activation of arylsulfatases, HSF1 activation, Serotonin Neurotransmitter Release Cycle, Dopamine Neurotransmitter Release Cycle, Attenuation phase, Receptor-type tyrosine-protein phosphatases, Ub-specific processing proteases, and Neuronal System. BP, biological process; CC, cellular component; MF, molecular function; GO, Gene Ontology; KEGG, Kyoto Encyclopedia of Genes and Genomes. 
A
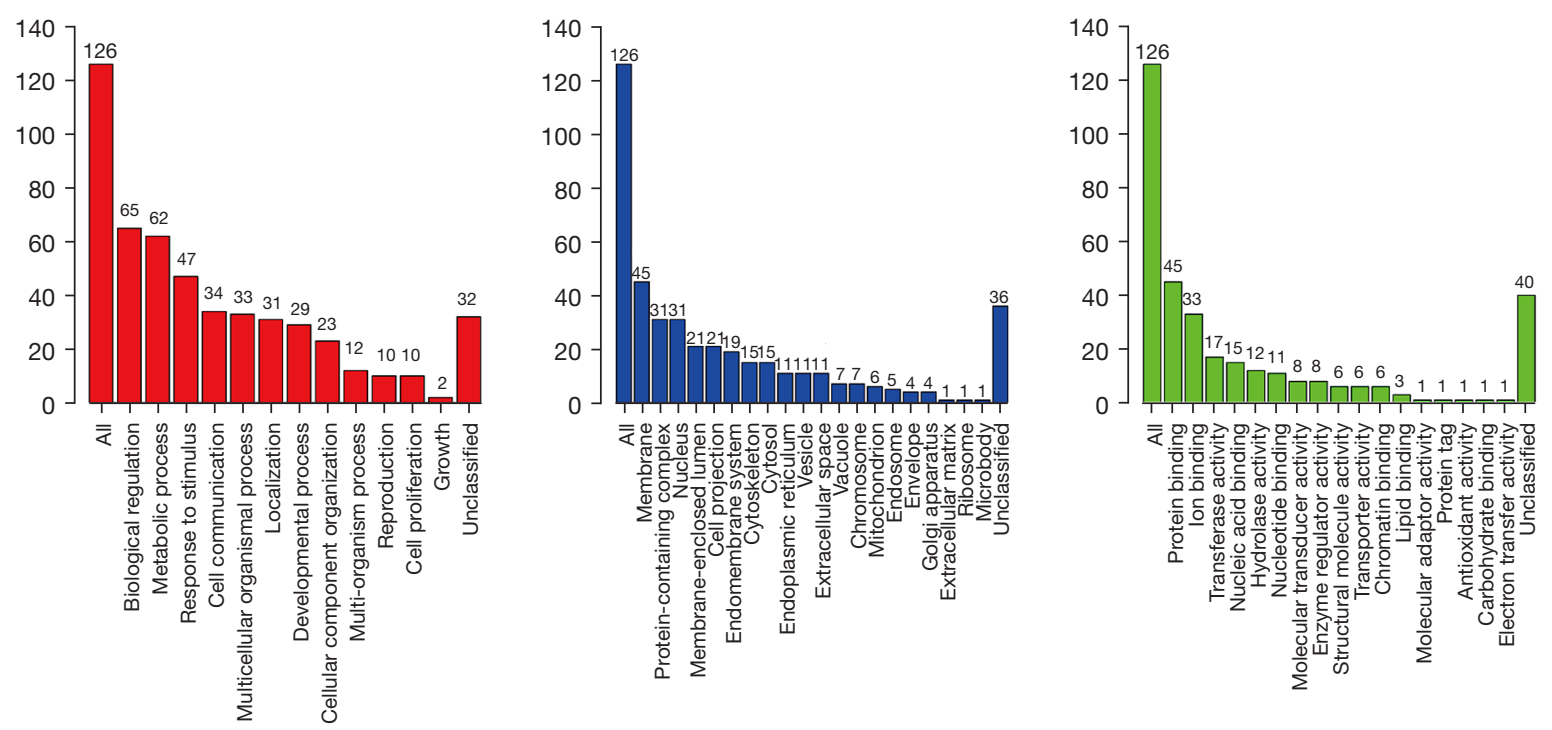

B

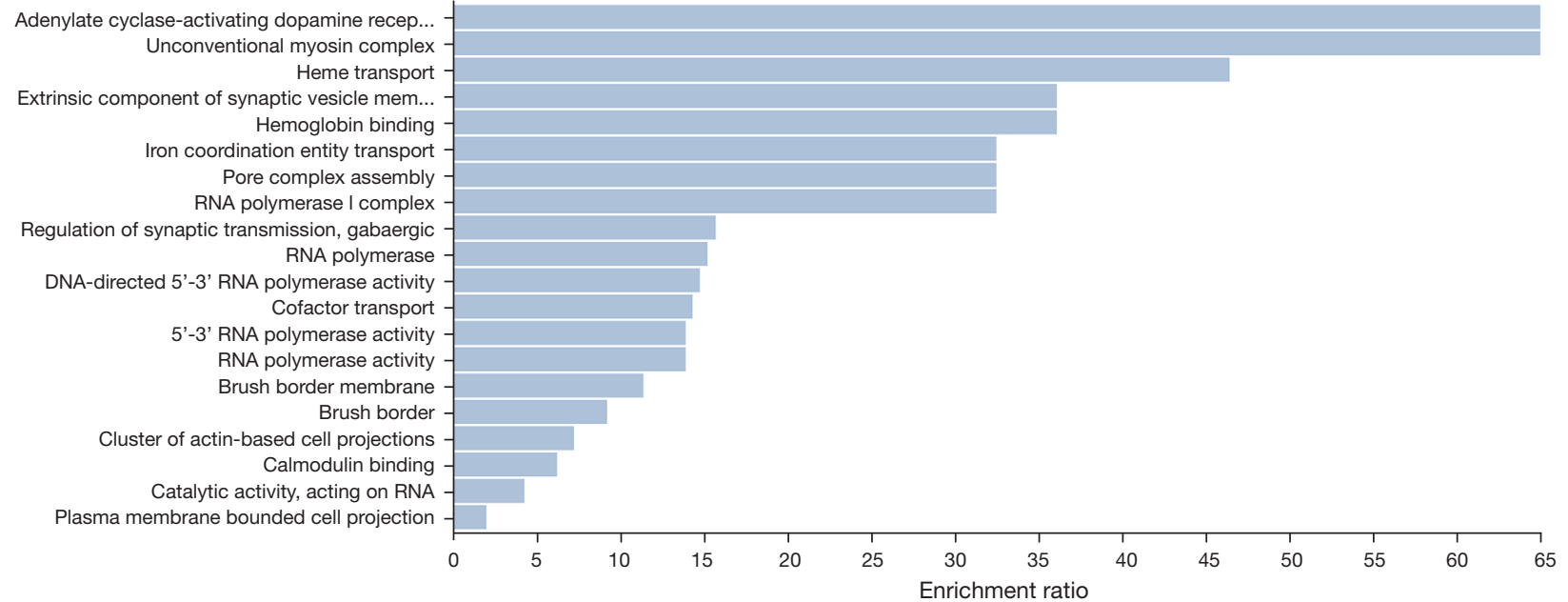

Figure 4 Enrichment analysis based on all common ROIs between 2 generations, (not confined to the $\mathrm{X}$ chromosome). There are 114 common ROIs (genes) in all chromosomes. (A) The GO summary for 3 categories; (B) all enriched GO terms, KEGG pathways, and reactome pathways (only one enriched KEGG pathway: RNA polymerase, and no reactome pathway). ROIs, regions of interest; GOI, Gene Ontology; KEGG, Kyoto Encyclopedia of Genes and Genomes.

seldom been investigated in PAH studies, and they can be used as valuable targets.

However, when we more strictly used false discovery rate $(\mathrm{FDR})<0.05$ as a standard, the above enrichments based on the X-chromosome ROIs and DEPs were not statistically significant. Therefore, we further observed all common ROIs and DEPs between 2 generations, which were not confined to the $\mathrm{X}$ chromosome. There were 114 common ROIs (genes) in all chromosomes. The GO summary for the 3 categories is represented in Figure 4A. All enriched GO terms, KEGG pathways, and reactome pathways are shown in Figure $4 B$ (only 1 enriched KEGG pathway: RNA polymerase was found, and no reactome pathway was found). Parallelly, there were 485 common DEPs (genes) in all chromosomes. In GO enrichments, a summary for 3 categories and 
A
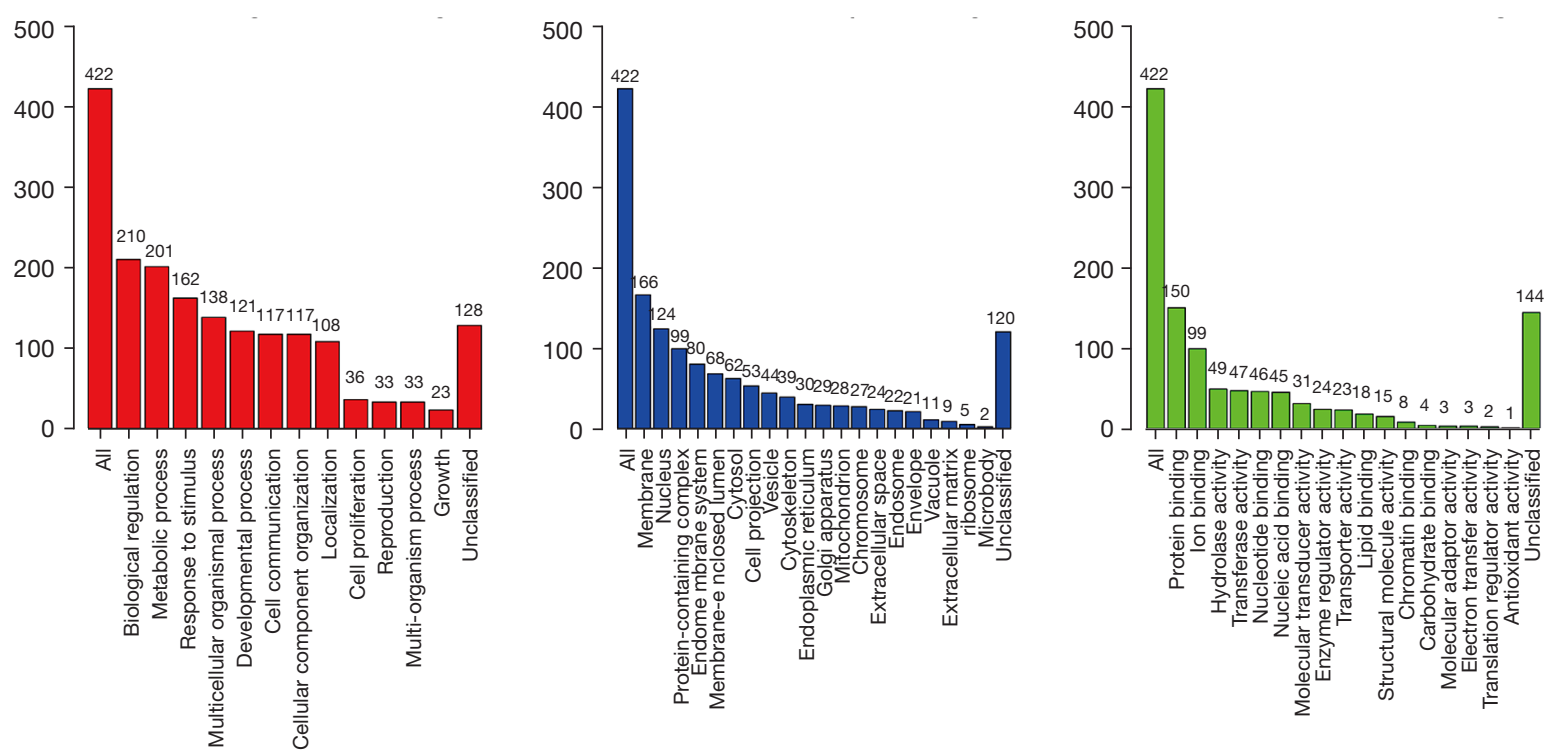

B

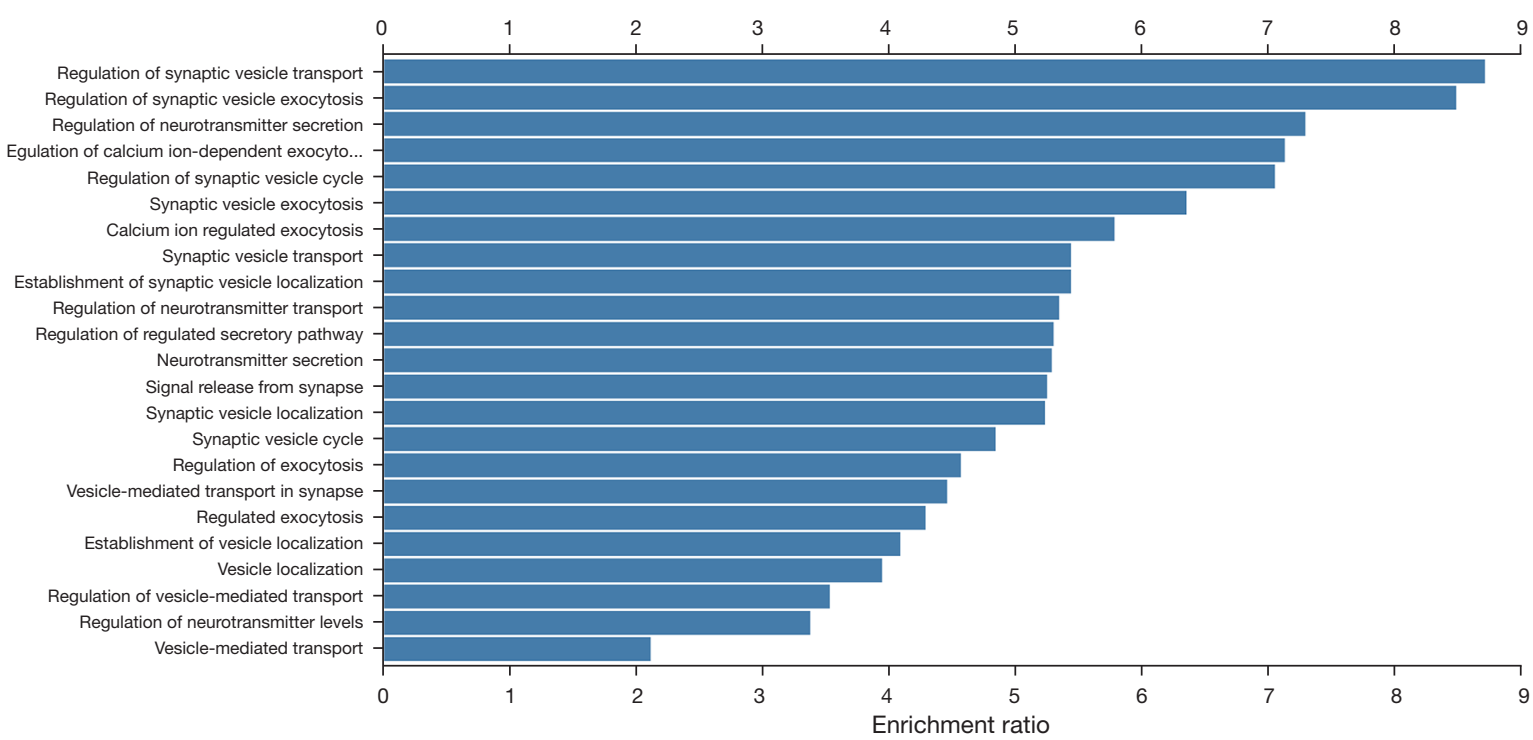

Figure 5 Enrichment analysis based on all common DEPs between 2 generations, (not confined to the X chromosome). There were 485 common DEPs (genes) in all chromosomes. (A) GO summary for 3 categories and the included genes; (B) all enriched GO terms, KEGG pathways, and reactome pathways. All the enriched terms are the BP category, such as regulation of synaptic vesicle transport, regulation of synaptic vesicle exocytosis, regulation of neurotransmitter secretion, regulation of calcium ion-dependent exocytosis, regulation of synaptic vesicle cycle, synaptic vesicle exocytosis, calcium ion regulated exocytosis, and synaptic vesicle transport establishment of synaptic vesicle localization. No other GO terms, KEGG pathways or reactome pathways are enriched. DEPs, differential enrichment peaks; GO, Gene Ontology; KEGG, Kyoto Encyclopedia of Genes and Genomes.

the included genes are represented in Figure $5 \mathrm{~A}$. Unlike the X-chromosome ROIs and DEPs or all ROIs, we noticed several significantly enriched (FDR $<0.05$ ) terms when using 485 common DEPs for enrichment analysis. All the enriched terms were the BP category
(Figure 5B), such as regulation of synaptic vesicle transport, regulation of synaptic vesicle exocytosis, regulation of neurotransmitter secretion, regulation of calcium iondependent exocytosis, regulation of synaptic vesicle cycle, synaptic vesicle exocytosis, calcium ion regulated exocytosis, 


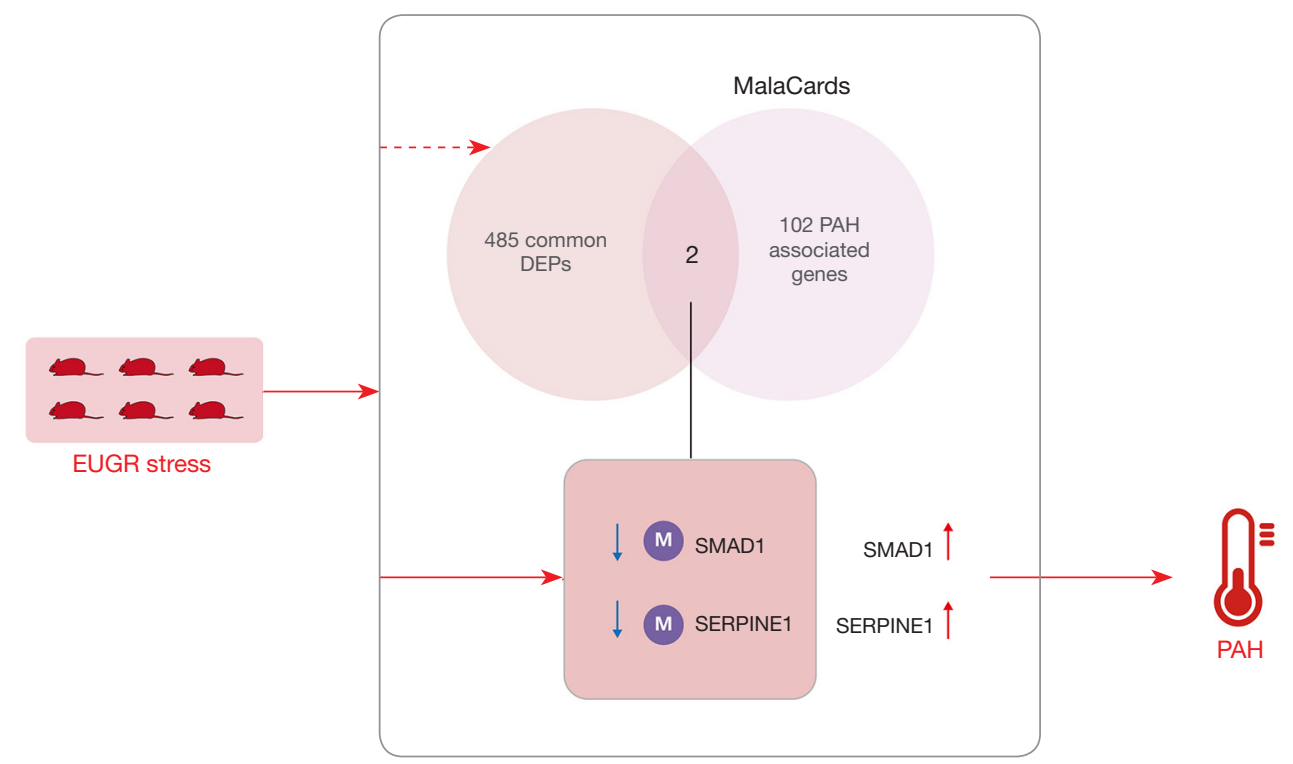

Figure 6 A possible mechanism of the EUGR-induced PAH development. There are 102 PAH-associated genes acquired from the MalaCards database and 485 common DEPs (between F1 and F2) in all chromosomes. Two common factors are found: Smad1 and Serpine1. Based on this finding, a potential mechanism of the EUGR-induced PAH development is that: under the EUGR stress, PVECs have impaired methylation of Smad1 and Serpine1, and an increased expression of SMAD1 and SERPINE1 partially triggers PAH in the later life. PAH, pulmonary arterial hypertension; EUGR, extrauterine growth restriction; DEPs, differential enrichment peaks; PVEC, pulmonary vascular endothelial cells.

and synaptic vesicle transport establishment of synaptic vesicle localization. No other GO terms, KEGG pathways, or reactome pathways were enriched. Together, synaptic and neurotransmitter relative pathways may be involved in the process of EUGR-induced PAH development. This novel insight needs more evidence to confirm.

Finally, due to a lack of validation experiments using the mRNA or protein expression, we checked the links between the 114 common ROIs/485 common DEPs in all chromosomes and $\mathrm{PAH}$-associated genes. There were $102 \mathrm{PAH}$-associated genes acquired from the MalaCards database (https://www.malacards.org/), and 2 common factors were found between 485 DEPs and the $102 \mathrm{PAH}-$ associated genes: Smad1 and Serpine1, which were both hypomethylation factors. Based on this finding, a potential mechanism of the EUGR-induced PAH development is depicted in Figure 6. Under the EUGR stress, PVECs have impaired methylation of Smad1 and Serpine1, and an increased expression of Smad1 and Serpine1 partially triggers $\mathrm{PAH}$ in later life.

\section{Discussion}

Early life stress such as physical abuse, trauma or neglect during a critical period of development can elicit negative long-lasting effects on health (11). Recently, it has been known that nutritional disadvantages during the early postnatal period may cause pulmonary vascular consequences in later life. For example, we have noticed that intrauterine growth retardation (IUGR) is associated with the development of adult-onset diseases, including pulmonary hypertension (12). The cblC defect tend to appear during infancy or early childhood and it may cause pulmonary hypertension in later life (13). Similarly, maternal undernutrition can induce differential cardiac gene expression in pulmonary hypertensive steers at high elevation (14). However, the clear mechanism underlying the development of pulmonary vascular driven by malnutrition is unknown. Epigenetic changes might mediate the cellular memory of early postpartum event. During PAH, the pulmonary artery undergoes chronic remodeling induced 
by constant proliferation of pulmonary endothelial cells and increased resistance to apoptosis which leads to an occlusion of the artery and subsequent pulmonary hypertension. It is not a surprise that chromatin remodeling has also been studied in the context of PAH (15). For example, HDAC class I activity was shown to increase during hypoxia induced but in vivo HDAC inhibition resulted in decreased pulmonary remodeling during PAH (16). Histone methylation has been implicated in PAH, where megakaryocytic leukemia 1 (MKL1) is upregulated and leads to an increase in the recruitment of the H3K4-specific complex components, ASH2 and WDR5 $(17,18)$. It has been known that epigenetic regulation by suv4-20h1 in cardiopulmonary progenitor cells is required to prevent pulmonary hypertension (19). Similarly, methylation and expression levels of the bmpr2 gene by $\sin 3 \mathrm{a}$ is a novel therapeutic mechanism in PAH (20). For the first time, this study verified that epigenetic dysregulation transmits susceptibility to $\mathrm{PAH}$ in the stressed generation and the next generation. In particular, we discovered 18 key candidate genes in chromosome X: Ptges 311, Rhox2, Slc7a3, Slitrk2, Syn1, Kcnd1, Tspyl2, Sts, Taf9b, Ngfrap1, Usp26, Kcne1l, RGD1565983, Tsx, RGD1562502, Bhlhb9, Bex2, and $M G C 114520$. The relationship of these potential targets to PAH is yet to be explored. Enriched pathways such as basal transcription factors and arachidonic acid metabolism, and reactome pathways like Phase-1-inactivation of fast $\mathrm{Na}+$ channels, aryl hydrocarbon receptor signaling, and activation of arylsulfatases may play essential roles. In all chromosomes, synaptic and neurotransmitter-associated biological processes are also important factors involved in EUGR-induced PAP disorders. Besides, attenuated methylation of Smad1 and Serpine1 in the promoter region may be a partial driver of PAH in later life.

Firstly, we believe that the above key candidates can be applied as potential targets in PAH research. However, none of these genes have been fully explored. Among these candidates, some interesting ones are worth surveying, especially synaptic and neurotransmitter relative factors. For example, the $S L C 7 A 3$ gene encodes a member of the solute carrier family 7 , a sodium-independent cationic amino acid transporter. It mediates the uptake of the cationic amino acids (arginine, lysine, and ornithine) in a sodium-independent manner. Upregulating Slc7a3, which causes an increase in arginine uptake, is involved in cancer cell adaptation to glutamine deprivation and the progression of papillary thyroid carcinoma $(21,22)$. The SLITRK2 gene encodes an integral membrane protein that contains $2 \mathrm{~N}$-terminal leucine-rich repeats domains and contains $\mathrm{C}$-terminal regions similar to neurotrophic receptors. The encoded protein may play a role in modulating neurite activity. It interacts directly with PSD-95 via a non-canonical Src homology 3 domainbinding motif that is associated with the $\mathrm{SH} 3$ domain of PSD-95 (23). Besides, the hyperactivity behavior that is modulated by Slitrk2 influences the excitatory and inhibitory synapse formation on dopamine neurons (24). The gene SYN1 is a member of the Synapsin gene family and is associated with the cytoplasmic surface of synaptic vesicles. It serves as a substrate for several different protein kinases, and phosphorylation may function in regulating this protein in the nerve terminal $(25,26)$. Mutations in this gene are perhaps associated with $\mathrm{X}$-linked disorders with primary neuronal degeneration, such as Rett syndrome. The epileptogenic SYN1 mutant may trigger an imbalance in release dynamics and short-term plasticity (27). This highlights that synaptic and neurotransmitter associated biological processes are essential factors involved in EUGR induced PAP disorders.

The EUGR environment may cause changes in nutritional factors [e.g., IGF2 (28)] and abnormal environment signals (29), which are the potential driving force of PAH. As we have mentioned above, Notch3 is important in mediating the nutritional signals, and it was found activated in smooth muscle cells, which promotes EUGR-induced pulmonary hypertension (30). Also, EUGR can promote the expression of inflammatory factors in the local microenvironment and the systematical physiological environment. The molecular mechanism may include IL-6, TNF- $\alpha$, IL-8, MCP-1, etc. $(31,32)$. Previously, PAH was known to be corrected with STAT3 (33), ALK5 (34), Gax (35), PDGF/PDGRR signaling (36,37), NF-kB signaling (38), HIF-1 $\alpha$ (8), Egr-1 (39), CD40 pathway (40), galectin-3 (41), SMAD3 (42), and a variety of micro-RNAs (43-46). At the epigenetic level, we have developed an IUGR and reported that the nutrient restriction increased the histone acetylation and HIF-1 $\alpha$ binding levels in the ET-1 gene promoter of PVEC in IUGR newborn rats, and this alteration continues up to 6 weeks after birth $(47,48)$. Additionally, known epigenetic alterations associated with PAH include BMPR, Endoglin, SMADs, Caveolin-1, $\mathrm{KCNK} 3$, and so on (49). Histone deacetylation (as well as histone deacetylase) plays a role in pulmonary artery smooth muscle cell hyperproliferation (10). Recently, increasing data have shed light on the epigenetic mechanisms underlying PAH (50). In the field of DNA methylation and PAH, the hypermethylation mechanisms in $\mathrm{CpG}$ islands mediated by 
DNMT1 and DNMT3B contribute to the downregulation of SOD 2 mRNA in PAH. These alterations may be able to enhance the proliferation of the pulmonary artery smooth muscle cells (PASMCs) in PAH (51).

However, very few studies have probed into the intergenerational inheritance of promoter methylation changes in PAP disorders induced by EUGR. In this study, we observed significant phenotype changes in F1 males and $\mathrm{F} 2$ females, which implied a pathway of epigenetic transmission through the $\mathrm{X}$ chromosome in male sperm. This finding is interesting and is consistent with some novel studies about the transgenerational epigenetic influences. Recently, different teams have paid attention on how impacts to the epigenetic information of parents affect offspring health (52). Human and animal studies have shown non-genomic transmission of programming effects of obesity or diabetes across generations, and the current mechanisms underlying either maternal or paternal influences on the metabolic status of offspring (53). Moreover, there are known transgenerational epigenetic influences of paternal environmental exposures on brain function and predisposition to psychiatric disorders (54). These changes can be mediated by germ cells in all possibility. It has been accepted that ionizing radiation may alter the epigenome of germ cells, leading to transgenerational reproductive impairments (55). A threegeneration study reported that exposures to tobacco smoke in men may cause lower lung function in future offspring, and this is completed by epigenetic changes transmitted through male germ cells (56). Our study may deepen the understanding of epigenetic transmission through the $\mathrm{X}$ chromosome in male sperm in the field of PAH and undernutrition.

Nevertheless, a limitation of this study is that we only investigated the relationship between DNA methylation and endothelial dysfunction, and not with sperm of F1 male model rats. In another work by our team, we established an IUGR model. Within ET-1 first intron, reduced DNA methylation and enhanced tri-methylation of lysine 4 on histone $\mathrm{H} 3$ were observed in PVECs and sperm of F1 generation of IUGR, with DNA demethylation in PVECs of F2 generation (57). More similar studies are to be conducted focusing on the transgenerational inheritance of promoter EUGR-induced PAP disorders.

Finally, after screening, we proposed that attenuated methylation of Smad1 and Serpine1 in the promoter region may be a partial driver of $\mathrm{PAH}$ in later life. In in the promoter region of the F1, the Peak-DM-Value of Smad1 was 0.238 and that of Serpine1 was 0.240. SMAD1 promoter hypermethylation and lack of SMAD1 expression were mainly related to Hodgkin lymphoma and bone morphogenetic protein signaling $(58,59)$. And Serpine1 promoter hypermethylation is associated with renal cell carcinoma, weight loss after laparoscopic sleeve gastrectomy, and inflammatory in macrophages (60-62). The attenuated methylation of Smad1 and Serpine1 in $\mathrm{PAH}$ has been never noticed as far as we known, and it is worth further research. However, a limitation of this study is that no mRNA and protein verifications were performed regarding the expression of SMAD1 and SERPINE1. In the future, this verification can be a priority for explanation of the detailed molecular mechanism of EUGR-induced PAH.

\section{Conclusions}

Together, there is transgenerational inheritance of promoter methylation changes in the $\mathrm{X}$ chromosome in EUGR induced PAP disorders. Besides, attenuated methylation of Smad1 and Serpine1 in the promoter region may be a partial driver of PAH in later life. Synaptic and neurotransmitter relative pathways are closely involved in this process.

\section{Acknowledgments}

Funding: This work was supported by the National Science Foundation for Young Scientists of China (81801497), Fuzhou Key Clinical Specialty Construction Project (201912007), and Fuzhou Science and Technology Plan Project (2019-SZ-54).

\section{Footnote}

Reporting Checklist: The authors have completed the ARRIVE reporting checklist. Available at https://dx.doi. org/10.21037/atm-21-4715

Data Sharing Statement: Available at https://dx.doi. org/10.21037/atm-21-4715

Conflicts of Interest: All authors have completed the ICMJE uniform disclosure form (available at https://dx.doi. org/10.21037/atm-21-4715). The authors have no conflicts of interest to declare.

Ethical Statement: The authors are accountable for all aspects of the work in ensuring that questions related 
to the accuracy or integrity of any part of the work are appropriately investigated and resolved. Experiments were performed under a project license (zju201305-1-02043) granted by The Affiliated Fuzhou Children Hospital of Fujian Medical University, in compliance with the institutional guidelines for the care and use of animals.

Open Access Statement: This is an Open Access article distributed in accordance with the Creative Commons Attribution-NonCommercial-NoDerivs 4.0 International License (CC BY-NC-ND 4.0), which permits the noncommercial replication and distribution of the article with the strict proviso that no changes or edits are made and the original work is properly cited (including links to both the formal publication through the relevant DOI and the license). See: https://creativecommons.org/licenses/by-nc-nd/4.0/.

\section{References}

1. Suresh K, Shimoda LA. Endothelial Cell Reactive Oxygen Species and Ca2+ Signaling in Pulmonary Hypertension. Adv Exp Med Biol 2017;967:299-314.

2. Zhu Y, Sun Y, Zhang S, et al. Xinmai 'an extract enhances the efficacy of sildenafil in the treatment of pulmonary arterial hypertension via inhibiting MAPK signalling pathway. Pharm Biol 2021;59:594-605.

3. Arai MA, Sakuraba K, Makita Y, et al. Evaluation of Naturally Occurring HIF-1 Inhibitors for Pulmonary Arterial Hypertension. Chembiochem 2021;22:2799-804.

4. Diebold I, Hennigs JK, Miyagawa K, et al. BMPR2 preserves mitochondrial function and DNA during reoxygenation to promote endothelial cell survival and reverse pulmonary hypertension. Cell Metab 2015;21:596-608.

5. Li Y, Chen X, Zeng X, et al. Galectin-3 mediates pulmonary vascular endothelial cell dynamics via TRPC1/4 under acute hypoxia. J Biochem Mol Toxicol 2020;34:e22463.

6. Watts JA, Marchick MR, Gellar MA, et al. Upregulation of arginase II contributes to pulmonary vascular endothelial cell dysfunction during experimental pulmonary embolism. Pulm Pharmacol Ther 2011;24:407-13.

7. Tang LL, Zhang LY, Lao LJ, et al. Epigenetics of Notch1 regulation in pulmonary microvascular rarefaction following extrauterine growth restriction. Respir Res 2015;16:66.

8. Yan J, Shen Y, Wang Y, et al. Increased expression of hypoxia-inducible factor-1 $\alpha$ in proliferating neointimal lesions in a rat model of pulmonary arterial hypertension. Am J Med Sci 2013;345:121-8.

9. Megalou AJ, Glava C, Oikonomidis DL, et al. Transforming growth factor- $\beta$ inhibition attenuates pulmonary arterial hypertension in rats. Int J Clin Exp Med 2010;3:332-40.

10. Huston JH, Ryan JJ. The emerging role of epigenetics in pulmonary arterial hypertension: an important avenue for clinical trials (2015 Grover Conference Series). Pulm Circ 2016;6:274-84.

11. Czarnabay D, Dalmago J, Martins AS, et al. Repeated three-hour maternal deprivation as a model of earlylife stress alters maternal behavior, olfactory learning and neural development. Neurobiol Learn Mem 2019;163:107040.

12. Fu LC, Lv Y, Zhong Y, et al. Tyrosine phosphorylation of $\mathrm{Kv} 1.5$ is upregulated in intrauterine growth retardation rats with exaggerated pulmonary hypertension. Braz J Med Biol Res 2017;50:e6237.

13. Iodice FG, Di Chiara L, Boenzi S, et al. Cobalamin C defect presenting with isolated pulmonary hypertension. Pediatrics 2013;132:e248-51.

14. Han H, Hansen TR, Berg B, et al. Maternal undernutrition induces differential cardiac gene expression in pulmonary hypertensive steers at high elevation. Am J Physiol Heart Circ Physiol 2008;295:H382-9.

15. Kocken JMM, da Costa Martins PA. Epigenetic Regulation of Pulmonary Arterial Hypertension-Induced Vascular and Right Ventricular Remodeling: New Opportunities? Int J Mol Sci 2020;21:8901.

16. Cavasin MA, Demos-Davies K, Horn TR, et al. Selective class I histone deacetylase inhibition suppresses hypoxiainduced cardiopulmonary remodeling through an antiproliferative mechanism. Circ Res 2012;110:739-48.

17. Chen D, Yang Y, Cheng X, et al. Megakaryocytic leukemia 1 directs a histone $\mathrm{H} 3$ lysine 4 methyltransferase complex to regulate hypoxic pulmonary hypertension. Hypertension 2015;65:821-33.

18. Yang $\mathrm{Y}$, Cheng $\mathrm{X}$, Tian W, et al. MRTF-A steers an epigenetic complex to activate endothelin-induced proinflammatory transcription in vascular smooth muscle cells. Nucleic Acids Res 2014;42:10460-72.

19. Qi H, Liu H, Pullamsetti SS, et al. Epigenetic Regulation by Suv4-20h1 in Cardiopulmonary Progenitor Cells Is Required to Prevent Pulmonary Hypertension and Chronic Obstructive Pulmonary Disease. Circulation 2021;144:1042-58. 
20. Bisserier M, Mathiyalagan P, Zhang S, et al. Regulation of the Methylation and Expression Levels of the BMPR2 Gene by SIN3a as a Novel Therapeutic Mechanism in Pulmonary Arterial Hypertension. Circulation 2021;144:52-73.

21. Lowman XH, Hanse EA, Yang Y, et al. p53 Promotes Cancer Cell Adaptation to Glutamine Deprivation by Upregulating Slc7a3 to Increase Arginine Uptake. Cell Rep 2019;26:3051-3060.e4.

22. Shen L, Qian C, Cao H, et al. Upregulation of the solute carrier family 7 genes is indicative of poor prognosis in papillary thyroid carcinoma. World J Surg Oncol 2018;16:235.

23. Loomis C, Stephens A, Janicot R, et al. Identification of MAGUK scaffold proteins as intracellular binding partners of synaptic adhesion protein Slitrk2. Mol Cell Neurosci 2020;103:103465.

24. Salesse C, Charest J, Doucet-Beaupré H, et al. Opposite Control of Excitatory and Inhibitory Synapse Formation by Slitrk2 and Slitrk 5 on Dopamine Neurons Modulates Hyperactivity Behavior. Cell Rep 2020;30:2374-2386.e5.

25. Guarnieri FC, Pozzi D, Raimondi A, et al. A novel SYN1 missense mutation in non-syndromic X-linked intellectual disability affects synaptic vesicle life cycle, clustering and mobility. Hum Mol Genet 2017;26:4699-714.

26. Fassio A, Patry L, Congia S, et al. SYN1 loss-of-function mutations in autism and partial epilepsy cause impaired synaptic function. Hum Mol Genet 2011;20:2297-307.

27. Lignani G, Raimondi A, Ferrea E, et al. Epileptogenic Q555X SYN1 mutant triggers imbalances in release dynamics and short-term plasticity. Hum Mol Genet 2013;22:2186-99.

28. Tozzi MG, Moscuzza F, Michelucci A, et al. ExtraUterine Growth Restriction (EUGR) in Preterm Infants: Growth Patterns, Nutrition, and Epigenetic Markers. A Pilot Study. Front Pediatr 2018;6:408.

29. Peila C, Spada E, Giuliani F, et al. Extrauterine Growth Restriction: Definitions and Predictability of Outcomes in a Cohort of Very Low Birth Weight Infants or Preterm Neonates. Nutrients 2020;12:1224.

30. Wang Y, Dai S, Cheng X, et al. Notch3 signaling activation in smooth muscle cells promotes extrauterine growth restriction-induced pulmonary hypertension. Nutr Metab Cardiovasc Dis 2019;29:639-51.

31. Mackay CA, Smit JS, Khan F, et al. IL-6 Predicts Poor Early Post-Natal Growth in Very Low-Birth-Weight Infants in a Low-Middle Income Setting. J Trop Pediatr 2021;67:fmaa132.
32. Ortiz-Espejo M, Pérez-Navero JL, Olza-Meneses J, et al. Prepubertal children with a history of extra-uterine growth restriction exhibit low-grade inflammation. Br J Nutr 2014;112:338-46.

33. Mukhopadhyay S, Shah M, Xu F, et al. Cytoplasmic provenance of STAT3 and PY-STAT3 in the endolysosomal compartments in pulmonary arterial endothelial and smooth muscle cells: implications in pulmonary arterial hypertension. Am J Physiol Lung Cell Mol Physiol 2008;294:L449-68.

34. Thomas M, Docx C, Holmes AM, et al. Activin-like kinase 5 (ALK5) mediates abnormal proliferation of vascular smooth muscle cells from patients with familial pulmonary arterial hypertension and is involved in the progression of experimental pulmonary arterial hypertension induced by monocrotaline. Am J Pathol 2009;174:380-9.

35. Xia S, Tai X, Wang Y, et al. Involvement of Gax gene in hypoxia-induced pulmonary hypertension, proliferation, and apoptosis of arterial smooth muscle cells. Am J Respir Cell Mol Biol 2011;44:66-73.

36. Xing AP, Hu XY, Shi YW, et al. Implication of PDGF signaling in cigarette smoke-induced pulmonary arterial hypertension in rat. Inhal Toxicol 2012;24:468-75.

37. Shaw DE, Baig F, Bruce I, et al. Optimization of PlateletDerived Growth Factor Receptor (PDGFR) Inhibitors for Duration of Action, as an Inhaled Therapy for Lung Remodeling in Pulmonary Arterial Hypertension. J Med Chem 2016;59:7901-14.

38. Hosokawa S, Haraguchi G, Sasaki A, et al. Pathophysiological roles of nuclear factor kappaB (NF$\mathrm{kB}$ ) in pulmonary arterial hypertension: effects of synthetic selective NF-kB inhibitor IMD-0354. Cardiovasc Res 2013;99:35-43.

39. Dickinson MG, Kowalski PS, Bartelds B, et al. A critical role for Egr-1 during vascular remodelling in pulmonary arterial hypertension. Cardiovasc Res 2014;103:573-84.

40. YanYun P, Wang S, Yang J, et al. Interruption of CD40 Pathway Improves Efficacy of Transplanted Endothelial Progenitor Cells in Monocrotaline Induced Pulmonary Arterial Hypertension. Cell Physiol Biochem 2015;36:683-96.

41. Wang X, Wang Y, Zhang J, et al. Galectin-3 contributes to vascular fibrosis in monocrotaline-induced pulmonary arterial hypertension rat model. J Biochem Mol Toxicol 2017.

42. Zabini D, Granton E, Hu Y, et al. Loss of SMAD3 Promotes Vascular Remodeling in Pulmonary Arterial Hypertension via MRTF Disinhibition. Am J Respir Crit Care Med 2018;197:244-60. 
43. Zhu G, Zhang W, Liu Y, et al. miR 371b 5p inhibits endothelial cell apoptosis in monocrotaline induced pulmonary arterial hypertension via PTEN/PI3K/Akt signaling pathways. Mol Med Rep 2018;18:5489-501.

44. Liu A, Liu Y, Li B, et al. Role of miR-223-3p in pulmonary arterial hypertension via targeting ITGB3 in the ECM pathway. Cell Prolif 2019;52:e12550.

45. Liu T, Zou XZ, Huang N, et al. miR-27a promotes endothelial-mesenchymal transition in hypoxia-induced pulmonary arterial hypertension by suppressing BMP signaling. Life Sci 2019;227:64-73.

46. Nie X, Chen Y, Tan J, et al. MicroRNA-221-3p promotes pulmonary artery smooth muscle cells proliferation by targeting AXIN2 during pulmonary arterial hypertension. Vascul Pharmacol 2019;116:24-35.

47. Xu XF, Cheng F, Du LZ. Epigenetic regulation of pulmonary arterial hypertension. Hypertens Res 2011;34:981-6.

48. Liu D, Morrell NW. Genetics and the molecular pathogenesis of pulmonary arterial hypertension. Curr Hypertens Rep 2013;15:632-7.

49. Kim JD, Lee A, Choi J, et al. Epigenetic modulation as a therapeutic approach for pulmonary arterial hypertension. Exp Mol Med 2015;47:e175.

50. Luna RCP, de Oliveira Y, Lisboa JVC, et al. Insights on the epigenetic mechanisms underlying pulmonary arterial hypertension. Braz J Med Biol Res 2018;51:e7437.

51. Archer SL. Acquired Mitochondrial Abnormalities, Including Epigenetic Inhibition of Superoxide Dismutase 2, in Pulmonary Hypertension and Cancer: Therapeutic Implications. Adv Exp Med Biol 2016;903:29-53.

52. Xavier MJ, Roman SD, Aitken RJ, et al. Transgenerational inheritance: how impacts to the epigenetic and genetic information of parents affect offspring health. Hum Reprod Update 2019;25:518-40.

53. Portha B, Grandjean V, Movassat J. Mother or Father: Who Is in the Front Line? Mechanisms Underlying the Non-Genomic Transmission of Obesity/Diabetes via the Maternal or the Paternal Line. Nutrients 2019;11:233.

Cite this article as: Tang L, Chen P, Yang L, Liu J, Zheng Y, Lin J, Chen S, Luo Y, Chen Y, Ma X, Zhang L. Transgenerational inheritance of promoter methylation changes in extrauterine growth restriction-induced pulmonary arterial pressure disorders. Ann Transl Med 2021;9(20):1551. doi: 10.21037/atm-21-4715
54. Yeshurun S, Hannan AJ. Transgenerational epigenetic influences of paternal environmental exposures on brain function and predisposition to psychiatric disorders. Mol Psychiatry 2019;24:536-48.

55. Leung CT, Yang Y, Yu KN, et al. Low-Dose Radiation Can Cause Epigenetic Alterations Associated With Impairments in Both Male and Female Reproductive Cells. Front Genet 2021;12:710143.

56. Accordini S, Calciano L, Johannessen A, et al. Prenatal and prepubertal exposures to tobacco smoke in men may cause lower lung function in future offspring: a three-generation study using a causal modelling approach. Eur Respir J 2021;58:2002791.

57. Zhang Z, Luo X, Lv Y, et al. Intrauterine Growth Restriction Programs Intergenerational Transmission of Pulmonary Arterial Hypertension and Endothelial Dysfunction via Sperm Epigenetic Modifications. Hypertension 2019;74:1160-71.

58. Gerlach MM, Stelling-Germani A, Ting Wu C, et al. SMAD1 promoter hypermethylation and lack of SMAD1 expression in Hodgkin lymphoma: a potential target for hypomethylating drug therapy. Haematologica 2021;106:619-21.

59. Gao S, Wang Z, Wang W, et al. The lysine methyltransferase SMYD2 methylates the kinase domain of type II receptor BMPR2 and stimulates bone morphogenetic protein signaling. J Biol Chem 2017;292:12702-12.

60. Sui Y, Lu K, Fu L. Prediction and analysis of novel key genes ITGAX, LAPTM5, SERPINE1 in clear cell renal cell carcinoma through bioinformatics analysis. PeerJ 2021;9:e11272.

61. Assem S, Abdelbaki TN, Mohy-El Dine SH, et al. SERPINE-1 Gene Methylation and Protein as Molecular Predictors of Laparoscopic Sleeve Gastrectomy Outcome. Obes Surg 2020;30:2620-30.

62. Samblas M, Martínez JA, Milagro F. Folic Acid Improves the Inflammatory Response in LPS-Activated THP-1 Macrophages. Mediators Inflamm 2018;2018:1312626. 